\title{
Fokussierte Prozessoptimierung im Spital
}

\author{
Dominik Mosera, Alfred Angerer ${ }^{b}$ \\ a Betriebsökonom, Fachmann Gesundheit, Spital Männedorf AG \\ b Prof. Dr. oec. HSG, Zürcher Hochschule für Angewandte Wissenschaften (ZHAW), Winterthur
}

\begin{abstract}
Das Lean Management feiert zurzeit grosse Erfolge beim Optimieren von Prozessen in Schweizer Krankenhäusern. Die für die Optimierung nötigen Ressourcen sind knapp bemessen, deswegen müssen die Initiativen fokussiert auf die Brennpunkte eines Spitals ausgerichtet sein. Dieser Beitrag zeigt, dass Spitäler sich vielfach auf die falschen Bereiche konzentrieren, und schlägt daher eine alternative Herangehensweise vor.
\end{abstract}

\section{Steigender Druck ist nicht gleich hoher Druck}

Immer anspruchsvollere Patienten verlangen höchste Behandlungsqualität; zugleich steigt der Kostendruck im Gesundheitswesen. Ineffizienzen im operativen Tagesgeschäft der Spitallandschaft zeugen davon, dass durchaus noch Geld und Verbesserungspotentiale vorhanden sind. Das haben einige Spitäler erkannt und gestalten ihre Prozessabläufe mit der Lean-Philosophie erfolgreich um, wie das kürzlich in der Ärztezeitung erschienene Round-Table-Gespräch zeigte (Sax, 2017). So eindrücklich und wichtig diese Einzelprojekte sind, so bleibt die Frage nach der Skalierung - schliesslich

\section{Résumé}

Quand les hôpitaux adoptent la philosophie Lean pour améliorer leurs procédures, ils le font souvent de façon déstructurée et ponctuelle, dans des services isolés, pour l'onéreuse procédure opératoire par exemple. Eliyahu Goldratt, à l'origine de la théorie des contraintes, recommande de considérer les organisations comme des systèmes, de rechercher leur goulet d'étranglement et d'améliorer les processus à ce niveau. A partir d'une étude de cas à l'hôpital de Männedorf, une chaîne de processus partiels a été examinée afin d'identifier le goulet d'étranglement. Pour ce faire, la procédure Hospital Bottleneck Process, décrivant la gestion du goulet d'étranglement à I'hôpital, a été mise au point. Cette analyse a permis de mettre en lumière la difficulté, l'idée étant de partir de ce constat pour améliorer les procédures de façon active et efficace, suivant la philosophie Lean. Pour terminer, les auteurs recommandent d'utiliser la théorie des contraintes de la même manière pour l'ensemble des prestations de santé de tous les établissements, afin d'améliorer notre système de santé de façon globale, en maîtrisant les coûts. soll das Gesamtsystem «Spital» verbessert werden. Aus Komplexitätsgründen fokussieren sich Lean-Projekte oft auf Prozesse innerhalb einzelner Abteilungen, wie eine Klinik-Sprechstunde oder Pflegestation (Sax, 2017). Dass Patientenpfade insgesamt optimiert werden, bleibt die seltene Ausnahme. Dieser Beitrag soll aufzeigen, wie Krankenhäuser von der Engpass-Theorie bei der Einführung von Lean profitieren würden, indem sie sich nämlich auf die Eliminierung des Engpasses (Bottleneck) fokussieren.

\section{Der traditionelle Lean-Transformation- Ansatz}

Typischerweise vermeidet man in schwer zu steuernden Expertenorganisationen wie Spitälern grosse Konflikte zwischen dem Management und dem medizinischen Personal. Deswegen gestalten die meisten Organisationen ihre Transformation in kleinen, sequentiellen Schritten, so auch die US-Pioniere wie das Virginia Mason oder Seattle Children's (Angerer, Brand u.a., 2016). Obwohl eine radikale Neugestaltung sämtlicher Prozesse nach der Lehre des Business Process Reengineering intellektuell sehr ansprechend ist, hat sich diese in der Spitalpraxis nur sehr selten durchsetzen können. Zu gross sind die Risiken und menschlichen Veränderungswiderstände bei der Umsetzung. Wenn Schweizer Spitäler die Lean-Reise beginnen, tappen Sie häufig in die Opportunismusfalle: Pilotprojekte, z.B. in der Logistik, zeigen substantielle Verbesserungen und erzeugen positive Veränderungsenergie im Unternehmen. Doch schon bei der Auswahl der nächsten Prozesse werden oft die falschen «Baustel- 
len» ausgesucht. Entweder werden Prozesse in Abteilungen bestimmt, bei denen man mit schwachem Widerstand der beteiligten Personen rechnet, oder es werden teure, prestigereiche Bereiche priorisiert (z.B. Operationsprozess). Dass Prozesse optimiert werden, ist an sich eine begrüssenswerte Angelegenheit. Noch effizienter wäre es allerdings, Prozesse anzugehen, die zur Verbesserung der Gesamtorganisation beitragen. Eine Lösung des Auswahlproblems könnte der Einbezug der Critical-Chain-Theorie darstellen. Diesen neuen Ansatz haben die Autoren dieses Artikels den «systemorientierten Pragmatismus» getauft.

\section{Herkunft des systemorientierten Pragmatismus}

Wir leben in einer Welt voller Systeme. Vielfach wird der Systembegriff mit der Informationstechnologie (IT) in Verbindung gebracht. Diese Betrachtungsweise greift jedoch deutlich zu kurz. Für Beer (1963; zitiert nach Haberfellner, Weck, Fricke, \& Vössner, 2012, S. 34) ist ein System «eine Ansammlung von miteinander in Beziehung stehender Teile [...], die in gewisser Hinsicht ein Ganzes bildet». Der Begriff umfasst damit nicht nur technische Apparate (wie ein Mobiltelefon), sondern auch biologische Systeme (wie der menschliche Körper) oder abstrakte, soziale Systeme. Gesundheitsorganisationen sind ebenfalls Systeme mit vielen Schnittstellen und somit inhärent komplex. Kybernetiker wie Beer, Malik und Probst gehen davon aus, dass Organisationen ähnlichen Entwicklungsprozessen und Wirkungsprinzipien ausgesetzt sind wie die Umwelt (Beer, 1963; Malik \& Probst, 1981). Entsprechend ist es sinnvoll, die Eigenschaften eines Systems als Spital zu kennen und bei der Prozessverbesserung zu berücksichtigen. So verfügt jedes System über einen Engpass, auch genannt Flaschenhals, der die Leistung des Gesamtsystems limitiert (Goldratt Institute, 2009; Techt \& Jakobi, 2010). Goldratt entwickelte auf Basis dieser Erkenntnis die Engpass-Theorie (Theory of Constraints): Das Ziel des Engpass-Managements ist, dass Entscheidungen auf lokaler Ebene (Bereich, Abteilung) im Sinne der Gesamtorganisation gefällt werden, und nicht abteilungsspezifisch Ergebnisse maximiert sowie Kosten

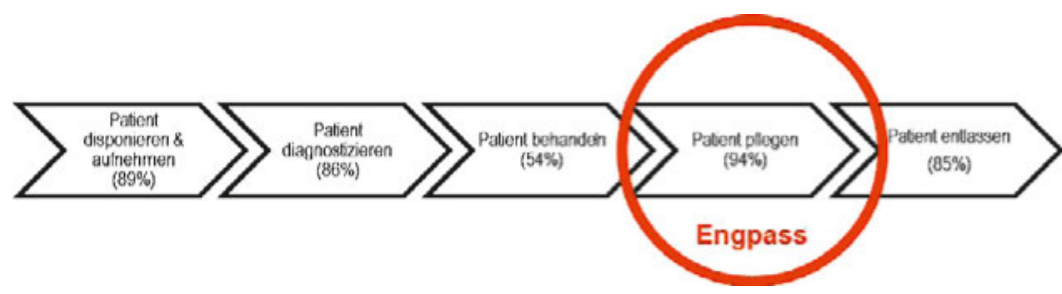

Abbildung 1: Beispiel-Spital mit Kapazitätsauslastung pro Prozessschritt (eigene Darstellung). minimiert werden. Nur die Verbesserung am Engpass ist betriebswirtschaftlich relevant.

Eine Kette ist so gut wie das schwächste Glied. Diese Erkenntnis der Systemtheorie gilt auch für Spitäler. Auf der Abbildung 1 wird eine vereinfachte Prozesskette eines Spitals dargestellt. Bei diesem Spital beträgt die Kapazitätsauslastung der Pflegestation 94\% und stellt somit den Systemengpass dar. Wenn das Gesamtsystem optimiert werden soll, muss daher die Prozessverbesserung bei diesem Prozessschritt beginnen. Eine Optimierung an einer anderen Stelle in diesem Spital wäre zunächst wirkungslos. Wenn die Kapazität der Pflege erhöht wird, steigt gleichzeitig die Kapazität des gesamten Behandlungsprozesses. Allerdings nur so lange, bis eine andere Stelle zum neuen Engpass wird (bei diesem Beispiel «Patient aufnehmen»). In den USA, Holland und Deutschland werden die Erkenntnisse der EngpassTheorie bereits im Gesundheitswesen erfolgreich angewendet - die Schweiz hat diesbezüglich Nachholbedarf (Röling, 2010; Schindler \& Ralfs, 2012).

Die Engpass-Theorie verfügt über verschiedene Werkzeuge zur Messung, Analyse und Optimierung von Prozessen wie die Durchsatz-Rechnung (Throughput Accounting) oder die Betriebssteuerung (Drum-BufferRope). Den Kern der Engpass-Theorie bilden die fünf Fokussierungsschritte: 1. Engpass des Systems erkennen, 2. Systemengpass voll auslasten, 3. restliche Prozesse dem Engpass unterordnen, 4. Engpass beheben und 5. Neubeginn beim ersten Schritt.

\section{Der Hospital Bottleneck Process}

Im Rahmen einer Fallstudie am Spital Männedorf wurde untersucht, wie die Engpass-Theorie die Einführung von Lean Management im Spital unterstützen kann. Dafür wurde der Hospital Bottleneck Process (Abb. 2) entwickelt und an einem vereinfachten System mit drei Prozessschritten erprobt: Patient operieren, Patient überwachen, Patient pflegen (Moser, 2015).

Häufig werden Abläufe mit Fokus auf den teuersten Prozessschritt optimiert. So auch im Spitalumfeld: Das fehlende Engpass-Verständnis in Spitälern zeigt sich häufig am Operationsbereich (OP). Da der OP-Betrieb oft der kostenintensivste Bereich eines Spitals ist, wird darauf ein besonderes Augenmerk gelegt (Rong, Vogt, \& Goudakos, 2012). So auch im Spital Männedorf. Da die Finanzergebnisse negativ ausfielen, mussten rasche Lösungen umgesetzt werden. Dank des Einsatzes von Belegärzten konnte die Anzahl an Behandlungen pro Tag in wenigen Monaten deutlich erhöht werden. Kurzfristig wurde damit eine überlebenswichtige Umsatzerhöhung erzielt. Inzwischen ist das OP-Team dabei, die Prozesse kontinuierlich zu optimieren. Aus 
Systemsicht brachte die Erhöhung der OP-Kapazität jedoch nicht die Erhöhung der Gesamtkapazität, denn der OP war nicht der Flaschenhals. Vor allem bei den Folgeprozessen in den Bettenstationen entstanden Schwierigkeiten, denn die Bettenstationen waren an vielen Tagen voll ausgelastet und stellten damit den wahren Flaschenhals dar. Entsprechend lohnt es sich, diesen Prozessschritt zu analysieren.

Der Hospital Bottleneck Process konnte im Spital Männedorf einerseits aufzeigen, dass die bestehende OP-Infrastruktur noch Potential zur besseren Auslastung hat; andererseits konnte mittels Datenanalyse

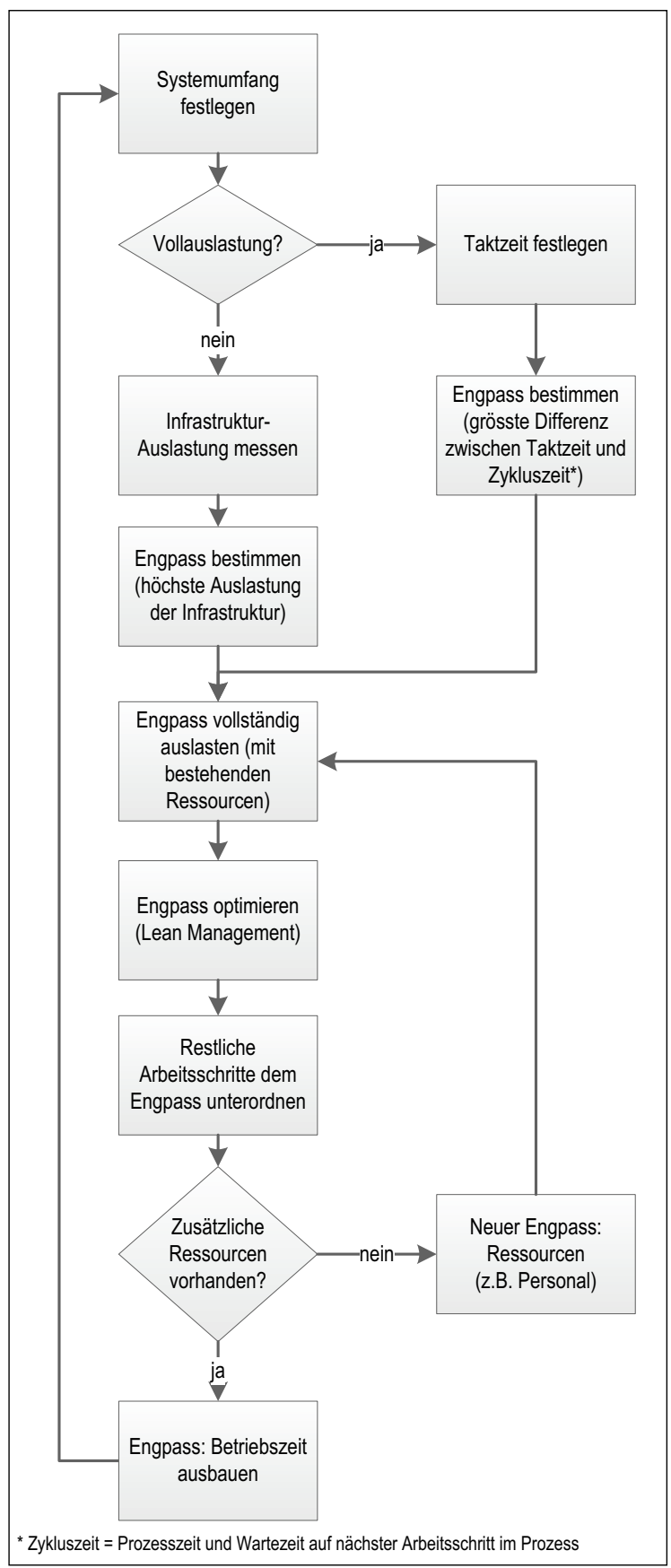

Abbildung 2: Hospital Bottleneck Process. belegt werden, dass der Engpass eindeutig beim Prozessschritt «Patient pflegen» vorliegt. Somit ist klar, dass die Bettenstationen in den primären Fokus gehören und nicht der OP.

\section{Fazit und Ausweitung der Kernideen auf die Health Value Chain}

Der Hospital Bottleneck Process hat für das System Spital seine Wirkung gezeigt. Der Ansatz verbindet Kernideen aus der Engpass-Theorie mit dem Lean Management. Anhand von Kapazitätsdaten können die Prozessverbesserungen fokussiert vorgenommen werden, um den Gesamtdurchsatz des Spitals zu maximieren. Weitere Case Studies sind in Zukunft notwendig, um den Ansatz zu validieren und zu verbessern.

Die Logik des Hospital Bottleneck Process kann zudem auf das gesamte Gesundheitssystem, die sogenannte Health Value Chain (Angerer, Liberatore u.a., 2016), ausgeweitet werden. So erweitern viele Gesundheitsbetriebe aktuell ihre Bettenkapazität. Da die Baukosten letztendlich vom Steuerzahler und den Bürgern getragen werden, darf dieser zwei Forderungen stellen: Einerseits müsste kritisch überprüft werden, ob die bestehende Infrastruktur bereits optimal ausgelastet wird; andererseits müsste die Health Value Chain grundsätzlich analysiert werden, um festzustellen, wo der tatsächliche Engpass vorliegt.

\section{Literatur}

- Angerer A, Brand T, Drews T, Hollenstein E, Liberatore F, Rüegg K, .. Vetterli C. (2016). LHT-BOK: Lean Healthcare Transformation Body of Knowledge. CreateSpace Independent Publishing Platform

- Angerer A, Liberatore F, Muschick I, Mussak P, Ruhse C, Schmidt R, ... Zeier A. (2016). Verbindung zweier Welten: Die Schnittstelle zwischen Spital und Krankenversicherer (S. 32). Zürich: Zürcher Hochschule für Angewandte Wissenschaften und Synpulse Schweiz AG.

- Beer S. (1963). Kybernetik und Management. Frankfurt: S. Fischer.

- Haberfellner R, Weck OL de, Fricke E \& Vössner S. (2012). Systems Engineering: Grundlagen und Anwendung. Zürich: Orell Füssli.

- Malik F, Probst G. (1981). Evolutionäres Management. Die Unternehmung, (35), 121-40.

- Moser D. (2015). Einführung von Prozessmanagement mittels Engpass-Theorie im Krankenhaus: Lean Management in der Spital Männedorf AG. Kalaidos Fachhochschule, Zürich

- Röling S. (2010). Mit TOC die Leistungsfähigkeit zuverlässig steigern - Erfahrungsbericht aus dem Groene Hart Krankenhaus in Gouda (NL). Das Krankenhaus, 102(3), 248.

- Rong O, Vogt A, Goudakos I. (2012). Baustelle OP - es gibt viel zu tun. Clinicum (4).

- Sax A. «Wo die Standardisierung aufhört, fängt das Denken an». Schweiz Ärztezeitung. 2017;98(6):191-4.

- Schindler WS, Ralfs D. (2012). Prozessoptimierung leicht gemacht: Vom klinischen Pfad zum kritischen Pfad Erfahrungsbericht zur Anwendung der Engpasstheorie in der AMEOS-Gruppe. Das Krankenhaus, 104(5), 488

Bildnachweise

Abbildung 1 und 2: Dominik Moser, 2015 